\title{
THE INTEGRAL FORMULA FOR THE REDUCED ALGEBRAIC MULTIPLICITY OF MEROMORPHIC OPERATOR FUNCTIONS
}

\author{
by H. BART, M. A. KAASHOEK and D. C. LAY
}

(Received 19th May 1976)

\section{Introduction}

Throughout this paper $A$ denotes an operator function, holomorphic on a deleted neighborhood of a complex number $\lambda_{o}$, with values in the space $\mathscr{L}(X, Y)$ of bounded linear operators between two complex Banach spaces $X$ and $Y$. In his survey article (7), I. C. Gohberg has defined for such an arbitrary operator function $A$ the algebraic multiplicity $M\left(A ; \lambda_{o}\right)$ and the reduced algebraic multiplicity $R M\left(A ; \lambda_{o}\right)$ of $A$ at $\lambda_{o}$. In earlier papers (e.g., $(8,16)$ ) these notions have been defined and studied for more restricted classes of operator functions. In (8) Gohberg and Sigal treated the case when $A$ is finite-meromorphic at $\lambda_{o}, A(\lambda)$ is bijective for $\lambda$ in some deleted neighborhood of $\lambda_{o}$ and the constant term $A_{0}$ in the Laurent expansion of $A$ at $\lambda_{o}$ is a Fredholm operator. They proved that in this case

$$
M\left(A ; \lambda_{o}\right)=\operatorname{tr}\left\{\frac{1}{2 \pi i} \int_{\Gamma} A^{\prime}(\lambda) A^{-1}(\lambda) d \lambda\right\}
$$

where $\Gamma$ is a suitable contour around $\lambda_{o}$. A first version of this formula was given by M. V. Keldyš (12) for the case of certain operator polynomials in Hilbert space (cf. 7, 8) and the references given there). In (16) Sigal has shown that in the non-bijective case (but $A_{0}$ still Fredholm)

$$
R M\left(A ; \lambda_{o}\right)=\operatorname{tr}\left\{\frac{1}{2 \pi i} \int_{\Gamma} A^{\prime}(\lambda) A^{+}(\lambda) d \lambda\right\}
$$

where $A^{+}$is a finite-meromorphic relative inverse of $A$, constructed in a rather special way. Further, $A^{+}$has the property that the associated projection functions $A^{+} A$ and $\boldsymbol{A A}^{+}$are holomorphic at $\lambda_{o}$. In Theorem 1, we extend Sigal's result to a wider class of operator functions. In fact, we show that $R M\left(A ; \lambda_{o}\right)$ is finite and formula (1) holds whenever $A$ is finite-meromorphic at $\lambda_{o}$ and $A^{+}$is any finite-meromorphic relative inverse of $A$ such that $A^{+} A$ and $A A^{+}$are holomorphic at $\lambda_{o}$. Theorem 1 applies to degenerate meromorphic operator functions as well as to finite-meromorphic operator functions with the property that the constant term in the Laurent expansion is a semi-Fredholm operator with complemented null space and range. Theorem 1 is used to prove that the reduced algebraic multiplicity, like the algebraic multiplicity (see $\mathbf{8}$, Theorem 5.2), has a certain logarithmic property (Theorem 2).

The authors wish to thank I. C. Gohberg for helpful remarks concerning the preparation of this revision of (6). 


\section{Preliminaries}

If $Z$ is a complex Banach space, then $\mathscr{H}\left(\lambda_{o}, Z\right)$ denotes the set of all $Z$-valued functions $\phi$ that are holomorphic on a deleted neighborhood of $\lambda_{o}$. If $\phi_{n}$ is the $n$-th coefficient in the Laurent expansion of $\phi$ at $\lambda_{o}$, then

$$
\nu\left(\phi ; \lambda_{o}\right)=\inf \left\{n: \phi_{n} \neq 0\right\} .
$$

Given $A$ in $\mathscr{H}\left(\lambda_{o}, \mathscr{L}(X, Y)\right)$ and an integer $m$, we let $H_{m}\left[A ; \lambda_{o}\right]$ be the set of all $x$ in $X$ for which there exists $\phi \in \mathscr{H}\left(\lambda_{o}, X\right)$ such that $\phi(\lambda) \rightarrow x$ as $\lambda \rightarrow \lambda_{o}$ and $\nu\left(A \phi ; \lambda_{o}\right) \geqslant$ $m+1$. Similarly $K_{m}\left[A ; \lambda_{o}\right]$ consists of all $y$ in $Y$ with the property that there exists $\phi \in \mathscr{H}\left(\lambda_{o}, X\right)$ such that $\nu\left(\phi ; \lambda_{o}\right) \geqslant-m$ and $A(\lambda) \phi(\lambda) \rightarrow y$ as $\lambda \rightarrow \lambda_{o}$. The intersection of the spaces $H_{m}\left[A ; \lambda_{o}\right]$ and the union of the spaces $K_{m}\left[A ; \lambda_{o}\right]$ are denoted by $H\left[A ; \lambda_{o}\right]$ and $K\left[A ; \lambda_{o}\right]$, respectively. The algebraic multiplicity $M\left(A ; \lambda_{o}\right)$ and the reduced algebraic multiplicity $R M\left(A ; \lambda_{o}\right)$ of $A$ at $\lambda_{o}$ are given by

$$
\begin{gathered}
M\left(A ; \lambda_{o}\right)=\sum_{m=0}^{\infty} \operatorname{dim} H_{m}\left[A ; \lambda_{o}\right]-\sum_{m=1}^{\infty} \operatorname{dim} K_{-m}\left[A ; \lambda_{o}\right] \\
R M\left(A ; \lambda_{o}\right)=\sum_{m=0}^{\infty} \operatorname{dim} \frac{H_{m}\left[A ; \lambda_{o}\right]}{H\left[A ; \lambda_{o}\right]}-\sum_{m=1}^{\infty} \operatorname{dim} K_{-m}\left[A ; \lambda_{o}\right]
\end{gathered}
$$

(whenever these formulas make sense). One verifies easily that these definitions are equivalent to those given by $I$. C. Gohberg in (7). His definitions are closer to the classical definition of the algebraic multiplicity of an eigenvalue of a single operator. The two sums in (2) are what Gohberg and Sigal have called the zero multiplicity and the pole multiplicity of $A$ at $\lambda_{o}$; the first term in the right hand side of (3) is what Sigal has called the reduced zero (or factor) multiplicity of $A$ at $\lambda_{o}$.

If $A$ is holomorphic at $\lambda_{o}$ (i.e., $\nu\left(A ; \lambda_{o}\right) \geqslant 0$ ), then the pole multiplicity of $A$ at $\lambda_{o}$ is zero (cf. 3, Proposition 1.3). Also, if $A$ is meromorphic at $\lambda_{o}$ (i.e., $\nu\left(A ; \lambda_{o}\right)>-\infty$ ), then the pole multiplicity of $A$ at $\lambda_{o}$ is finite if and only if $A$ is finite-meromorphic at $\lambda_{o}$ (cf. 3, Propositions 1.3 and 3.3). Finally, it is clear from the definitions that the reduced zero multiplicity of $A$ at $\lambda_{o}$ is finite if and only if the dimension of the quotient space $H_{0}\left[A ; \lambda_{o}\right] / H\left[A ; \lambda_{o}\right]$ is finite. This number plays an important role in $(3,5)$ and is called the stability number of $A$ at $\lambda_{o}$.

Given $A$ in $\mathscr{H}\left(\lambda_{o}, \mathscr{L}(X, Y)\right)$, an operator function $A^{+}$in $\mathscr{H}\left(\lambda_{o}, \mathscr{L}(Y, X)\right)$ is said to be a relative inverse of $A$ near $\lambda_{o}$ if, for $\lambda$ in some deleted neighborhood of $\lambda_{o}$,

$$
A(\lambda)=A(\lambda) A^{+}(\lambda) A(\lambda), \quad A^{+}(\lambda)=A^{+}(\lambda) A(\lambda) A^{+}(\lambda) .
$$

If $A^{+}$is a relative inverse of $A$ near $\lambda_{o}$, the functions $P$ and $Q$ given by $P(\lambda)=$ $A^{+}(\lambda) A(\lambda)$ and $Q(\lambda)=A(\lambda) A^{+}(\lambda)$ are called the projection functions associated with $A$ and $A^{+}$. Obviously $P$ and $Q$ are projection valued and holomorphic on a deleted neighborhood of $\lambda_{o}$. When $P$ and $Q$ are holomorphic at $\lambda_{o}$, then in many respects the behaviour of $A$ at $\lambda_{o}$ is determined by that of $A^{+}$and conversely (cf. 4, 5, 6).

\section{Main results}

For an arbitrary degenerate operator $F$, we let tr $F$ denote the trace of $F$ (cf. (11), Section III. 4.3). 
Theorem 1. Let $A$ be finite-meromorphic at $\lambda_{o}$, and suppose that there exists a finite-meromorphic relative inverse $A^{+}$of $A$ near $\lambda_{o}$ such that the associated projection functions are holomorphic at $\lambda_{o}$. Then $\operatorname{RM}\left(A ; \lambda_{o}\right)$ is finite and

$$
R M\left(A ; \lambda_{o}\right)=\operatorname{tr}\left\{\frac{1}{2 \pi i} \int_{\Gamma} A^{\prime}(\lambda) A^{+}(\lambda) d \lambda\right\}
$$

where $\Gamma$ is a sufficiently small circle centred at $\lambda_{o}$.

Proof. We use the reduction method described in Subsection 1.5 of (5). Let $P$ and $Q$ be the projection functions associated with $A$ and $A^{+}$. Then $P$ and $Q$ are holomorphic on an open neighborhood $U$ of $\lambda_{o}$ and for $\lambda_{o} \neq \lambda \in U$ the operator $P(\lambda)$ is a projection of $X$ along $N(A(\lambda))$ and $Q(\lambda)$ is a projection of $Y$ onto $R(A(\lambda))$. Define

$$
\begin{aligned}
& E(\lambda)=P\left(\lambda_{o}\right) P(\lambda)+\left(I_{X}-P\left(\lambda_{o}\right)\right)\left(I_{X}-P(\lambda)\right), \\
& F(\lambda)=Q\left(\lambda_{o}\right) Q(\lambda)+\left(I_{Y}-Q\left(\lambda_{o}\right)\right)\left(I_{Y}-Q(\lambda)\right) .
\end{aligned}
$$

Then $E(\lambda)$ and $F(\lambda)$ are bijective for $\lambda$ in some neighborhood $V$ of $\lambda_{o}$. For $\lambda_{o} \neq \lambda \in V$, put

$$
B(\lambda)=F(\lambda) A(\lambda) E^{-1}(\lambda), \quad B^{+}(\lambda)=E(\lambda) A^{+}(\lambda) F^{-1}(\lambda) .
$$

Then $N(B(\lambda))=N\left(P\left(\lambda_{o}\right)\right)$ and $R(B(\lambda))=R\left(Q\left(\lambda_{o}\right)\right)$. With respect to the decompositions of $X$ and $Y$ associated with the projections $P\left(\lambda_{o}\right)$ and $Q\left(\lambda_{o}\right)$, one may write

$$
B(\lambda)=0 \oplus C(\lambda), \quad \lambda_{o} \neq \lambda \in V .
$$

Here $C(\lambda)$ is the restriction of $B(\lambda)$ to $R\left(P\left(\lambda_{o}\right)\right)$ considered as an operator into $R\left(Q\left(\lambda_{o}\right)\right)$. Observe that $C(\lambda)$ is bijective. Also, one can show that

$$
B^{+}(\lambda)=0 \oplus C^{-1}(\lambda), \quad \lambda_{o} \neq \lambda \in V .
$$

By (5), Theorem 1.10 and Proposition 1.7, the conditions on $A$ imply that $C$ is finite-meromorphic at $\lambda_{o}$ and the constant term in the Laurent expansion of $C$ at $\lambda_{o}$ is Fredholm. But then one can apply the theory developed in (8) to show that $M\left(C ; \lambda_{o}\right)$ is finite and

$$
M\left(C ; \lambda_{o}\right)=\operatorname{tr}\left\{\frac{1}{2 \pi i} \int_{\Gamma} C^{\prime}(\lambda) C^{-1}(\lambda) d \lambda\right\}
$$

for some sufficiently small circle $\Gamma$ centred at $\lambda_{o}$. Observe that $B^{+}$is meromorphic at $\lambda_{o}$. It follows from formula (6) that $C^{-1}$ has the same property. Hence $H\left[C ; \lambda_{o}\right]=(0)$ by (2), Theorem 2.4. But then (5), Lemma 1.6 implies that $R M\left(A ; \lambda_{o}\right)=M\left(C ; \lambda_{o}\right)$. Using formulas (5) and (6) and the definition of the trace it is easy to show that

$$
\operatorname{tr}\left\{\frac{1}{2 \pi i} \int_{\Gamma} C^{\prime}(\lambda) C^{-1}(\lambda) d \lambda\right\}=\operatorname{tr}\left\{\frac{1}{2 \pi i} \int_{\Gamma} B^{\prime}(\lambda) B^{+}(\lambda) d \lambda\right\} .
$$

It remains to prove that the right hand sides of (4) and (7) are equal. For this purpose we employ a slight modification of an argument used in (16), Section 4 (cf. 8, Section 4). Omitting the variable $\lambda$, we have

$$
A^{\prime} A^{+}=F^{-1} B E^{\prime} E^{-1} B^{+} F+F^{-1} B^{\prime} B^{+} F+\left(F^{-1}\right)^{\prime} B B^{+} F \text {. }
$$


All three terms in the right hand side of this equality are finite-meromorphic at $\lambda_{o}$ and, in fact, the last one is holomorphic at $\lambda_{o}$. Using the properties of the trace and comparing Laurent expansions, one can easily prove that for finite-meromorphic operator functions $H$ and $J$ one has

$$
\operatorname{tr}\left\{\frac{1}{2 \pi i} \int_{\Gamma} H(\lambda) J(\lambda) d \lambda\right\}=\operatorname{tr}\left\{\frac{1}{2 \pi i} \int_{\Gamma} J(\lambda) H(\lambda) d \lambda\right\}
$$

(cf. 8). Thus we obtain

$$
\operatorname{tr}\left\{\frac{1}{2 \pi i} \int_{\Gamma} A^{\prime} A^{+} d \lambda\right\}=\operatorname{tr}\left\{\frac{1}{2 \pi i} \int_{\Gamma} E^{\prime} E^{-1} B^{+} B d \lambda\right\}+\operatorname{tr}\left\{\frac{1}{2 \pi i} \int_{\Gamma} B^{\prime} B^{+} d \lambda\right\} .
$$

Now the first integral in the right hand side is zero because the integrand is holomorphic at $\lambda_{o}$, and so the proof is complete.

An operator function $A$ will be called block-diagonable at $\lambda_{o}$ if $A$ satisfies the conditions of Theorem 1. To justify this terminology we mention that $A$ satisfies the conditions of Theorem 1 if and only if $A$ is equivalent at $\lambda_{o}$ in the sense of (7) to a (block-)diagonable operator function $D$ of the following type:

$$
D(\lambda)=\sum_{i=r}^{s}\left(\lambda-\lambda_{o}\right)^{i} Q_{i} D_{i} P_{i}
$$

where $r$ and $s$ are integers, $r \leqq 0<s$, and

(1) $\left\{P_{r}, \ldots, P_{s}\right\}$ and $\left\{Q_{r}, \ldots, Q_{s}\right\}$ are sets of mutually disjoint projections of $X$ and $Y$, respectively,

(2) $D_{i}$ is a bijective operator in $\mathscr{L}\left(X_{i}, Y_{i}\right)$, where $X_{i}=R\left(P_{i}\right)$ and $Y_{i}=R\left(Q_{i}\right)$, $i=r, \ldots, s$,

(3) $\operatorname{dim} R\left(P_{i}\right)<\infty$ for $i \neq 0$.

For details see (8), Theorem 3.1; (4), Section 4; or (5), last paragraph of Section 1. The class of block-diagonable operator functions includes all degenerate meromorphic operator functions and also all finite-meromorphic operator functions with the property that the constant term in the Laurent expansion is a semi-Fredholm operator with complemented null space and range. This is immediate from (5), Theorem 1.10 and the results of (3), Sections 6 and 7 .

If $A$ is block-diagonable at $\lambda_{o}$, and $A^{+}$is any relative inverse of $A$ near $\lambda_{o}$ with holomorphic associated projection functions, then $A^{+}$is automatically finitemeromorphic at $\lambda_{o}$ (cf. 5, Theorems 1.10 and 2.1). This fact will be used in the proof of the next theorem.

Theorem 2. Let $A \in \mathscr{H}\left(\lambda_{o}, \mathscr{L}(X, Y)\right)$ and $B \in \mathscr{H}\left(\lambda_{o}, \mathscr{L}(Z, X)\right)$, where $X, Y$ and $Z$ are complex Banach spaces. Suppose that $A$ and $B$ are block-diagonable at $\lambda_{o}$ and that

$$
X=H\left[A ; \lambda_{o}\right] \oplus K\left[B ; \lambda_{o}\right]
$$

Then $A B$ is block-diagonable at $\lambda_{o}$ and

$$
R M\left(A B ; \lambda_{o}\right)=R M\left(A ; \lambda_{o}\right)+R M\left(B ; \lambda_{o}\right) .
$$


Proof. From (3), Section 6 and (5), Section 1 we know that there exist projection functions $P_{A}, P_{B}, Q_{A}$ and $Q_{B}$, holomorphic at $\lambda_{o}$, such that

$$
\begin{array}{ll}
N\left(P_{A}(\lambda)\right)=H[A ; \lambda], & N\left(P_{B}(\lambda)\right)=H[B ; \lambda], \\
R\left(Q_{A}(\lambda)\right)=K[A ; \lambda], & R\left(Q_{B}(\lambda)\right)=K[B ; \lambda],
\end{array}
$$

for $\lambda$ in some neighborhood of $\lambda_{o}$. The condition expressed in formula (9) implies that $P_{A}$ and $Q_{B}$ may be taken to be equal. A proof of this is sketched in (14).

For $\lambda$ in a deleted neighborhood of $\lambda_{o}$, we have

$$
\begin{aligned}
H[A ; \lambda] & =N(A(\lambda)), & H[B ; \lambda] & =N(B(\lambda)), \\
K[A ; \lambda] & =R(A(\lambda)), & K[B ; \lambda] & =R(B(\lambda)) .
\end{aligned}
$$

Using the proof of (1), Theorem 1.3 one can establish the existence of relative inverses $A^{+}$and $B^{+}$of $A$ and $B$ near $\lambda_{o}$ such that (omitting the variable $\lambda$ )

$$
A A^{+}=Q_{A}, \quad A^{+} A=P_{A}=Q_{B}=B B^{+}, \quad B^{+} B=P_{B} .
$$

Since $A$ and $B$ are block-diagonable at $\lambda_{o}$, the functions $A^{+}$and $B^{+}$are both finitemeromorphic at $\lambda_{\sigma}$. Thus the same is true for their product $B^{+} A^{+}$. A straightforward computation shows that $B^{+} A^{+}$is a relative inverse of $A B$ near $\lambda_{o}$ with associated projection functions $P_{B}$ and $Q_{A}$. Hence $A B$ is block-diagonable at $\lambda_{0}$. It remains to show that $(10)$ is satisfied.

By Theorem 1 we have, for some sufficiently small circle $\Gamma$ centred at $\lambda_{o}$,

$$
\begin{aligned}
R M\left(A B ; \lambda_{o}\right) & =\operatorname{tr}\left\{\frac{1}{2 \pi i} \int_{\Gamma}(A B)^{\prime} B^{+} A^{+} d \lambda\right\} \\
& =\operatorname{tr}\left\{\frac{1}{2 \pi i} \int_{\Gamma} A^{\prime} B B^{+} A^{+} d \lambda\right\}+\operatorname{tr}\left\{\frac{1}{2 \pi i} \int_{\Gamma} A B^{\prime} B^{+} A^{+} d \lambda\right\}
\end{aligned}
$$

Using $B B^{+} A^{+}=A^{+}$and $B^{+} A^{+} A=B^{+}$together with formula (8) and Theorem 1 , one obtains the desired result.

Theorem 2 is a generalisation of earlier results which have appeared in $(8,13,15)$. In all these results, it was assumed that $A(\lambda)$ and $B(\lambda)$ are bijective for $\lambda$ in some deleted neighborhood of $\lambda_{o}$, whereas our theorem also applies, for example, when $A(\lambda)$ is injective and $B(\lambda)$ is surjective for $\lambda$ near $\lambda_{o}$ (for in this case results in $(3,4,5$ ) imply that $H\left[A ; \lambda_{o}\right]=(0)$ and $\left.K\left[B ; \lambda_{o}\right]=X\right)$. We conclude this paper with some examples which suggest that Theorem 2 cannot be generalised much further.

Let $P$ be a projection of rank one of a complex Banach space $Y$ and let $X=R(I-P)$. For $\lambda \in C$, let $B(\lambda)$ be the injection of $X$ into $Y$. Choose an integer $k$ and, for $\lambda \neq 0$, let

$$
A(\lambda)=I-P+\left(\lambda-\lambda_{o}\right)^{k} P
$$

Then $A$ and $B$ are block-diagonable at $\lambda_{o}$ and $H\left[A ; \lambda_{o}\right]=(0)$. Observe that $A(\lambda) B(\lambda)=I-P$, and so $R M\left(A B ; \lambda_{o}\right)=0$. However $R M\left(A ; \lambda_{o}\right)=k$ and $R M\left(B ; \lambda_{o}\right)=0$. This shows that in Theorem 2 the hypothesis (9) cannot be replaced by $H\left[A ; \lambda_{o}\right] \cap K\left[B ; \lambda_{o}\right]=(0)$. A similar example shows that (9) cannot be replaced by $H\left[A ; \lambda_{o}\right]+K\left[B ; \lambda_{o}\right]=X$. 
In (9) Gohberg and Sigal considered products of block-diagonable operator functions whose values are Fredholm operators. For the holomorphic case, Theorem 1 in their paper states that

$$
R M\left(A B ; \lambda_{o}\right) \leqslant R M\left(A ; \lambda_{o}\right)+R M\left(B ; \lambda_{o}\right) .
$$

This, however, is not correct. For instance, define $A(\lambda)$ and $B(\lambda)$ on $C^{2}$ by

$$
A(\lambda)=\left(\begin{array}{cc}
\lambda^{k} & 1 \\
0 & 0
\end{array}\right), \quad B(\lambda)=\left(\begin{array}{ll}
1 & 0 \\
0 & 0
\end{array}\right)
$$

where $k$ is a positive integer. Then $R M(A ; 0)=R M(B ; 0)=0$, whereas $R M(A B ; 0)=$ $k$. With minor modifications the proof in (9) holds for the case when $A(\lambda)$ is injective and $B(\lambda)$ is surjective for $\lambda$ near $\lambda_{o}$.

\section{REFERENCES}

(1) H. BART, Holomorphic relative inverses of operator valued functions, Math. Ann. 208 (1974), 179-194.

(2) H. B ART, Poles of the resolvent of an operator function, Proc. Royal Irish Acad. 74A (1974), 169-184.

(3) H. BART, M. A. KAASHOEK and D. C. LAY, Stability properties of finite meromorphic operator functions, Nederl. Akad. Wetensch. Proc. Ser. A 77 (1974), 217-259.

(4) H. BART, M. A. KAASHOEK and D. C. LAY, Relative inverses of meromorphic operator functions, University of Maryland Technical Report, TR-74-71, 1974.

(5) H. BART, M. A. KAASHOEK and D. C. LAY, Relative inverses of meromorphic operator functions and associated holomorphic projection functions, Math. Ann. 218 (1975), 199-210.

(6) H. BART, M. A. KAASHOEK and D. C. LAY, Reduced algebraic multiplicity of operator functions, University of Maryland Technical Report, TR-74-56, 1974.

(7) I. C. GoHBERG, On some topics of spectral theory of finitely meromorphic operator functions, Izv. Akad. Nauk. Armjan SSR Ser. Mat. 6 (1971), 160-181. [Russian]

(8) I. C. GoHBERg and E. I. SigAl, An operator generalisation of the logarithmic residue theorem and the theorem of Rouché, Mat. Sb.84 (126) (1971), 607-629 [Russian] = Math. USSR Sbornik 13 (1971), 603-625.

(9) I. C. GoHBERG and E. I. SIGAL, On the zero multiplicity of the product of meromorphic operator functions, Mat. Issled. 6 (1971), No. 2 (20), 33-50. [Russian]

(10) M. A. KaAshoek, Ascent, descent, nullity and defect, a note on a paper by A. E. Taylor, Math. Ann. 172 (1967), 105-115.

(11) T. KATo, Perturbation theory for linear operators, (Springer, Berlin-New York, 1966).

(12) M. V. KELDYS, On the eigenvalues and eigenfunctions of certain classes of nonselfadjoint equations, Dokl. Akad. Nauk SSSR 77 (1) (1951), 11-14. [Russian]

(13) A. S. MARkuS and E. I. Sigal, On the multiplicity of a characteristic value of an analytic operator-valued function, Mat. Issled. 5 (1970), No. 3 (17), 129-147. [Russian]

(14) M. A. ShuBIN, On holomorphic families of subspaces of a Banach space, Mat. Issled. 5 (1970), No. 4 (18), 153-165; Letter to the editors, Mat. Issled. 6 (1971), No. 1 (19), 180. [Russian]

(15) E. I. SIGAL, On the multiplicity of a characteristic value of a product of operatorvalued functions, Mat. Issled. 5 (1970), No. 1 (15), 118-127. [Russian] 
INTEGRAL FORMULA OF MEROMORPHIC OPERATOR FUNCTIONS 71

(16) E. I. SIGAL, Factor-multiplicity of eigenvalues of meromorphic operator functions, Mat. Issled. 5 (1970), No. 4 (18), 136-152. [Russian]

H. B ART AND M. A. KaASHOEK

Wiskundig Seminarium

VRIJE UNIVERSITEIT

AMSTERDAM-11
D. C. LAY

Department of Mathematics

UNIVERSITY OF MARYLAND

COllege Park, MD. 20742 